\title{
Physical activity, diet and gene-environment interactions in relation to body mass index and waist circumference: The Swedish Young Male Twins Study
}

\author{
Nina Karnehed ${ }^{1}$, Per Tynelius ${ }^{2}$, Berit L Heitmann ${ }^{3}$ and Finn Rasmussen ${ }^{1,2, *}$ \\ ${ }^{1}$ Child and Adolescent Public Health Epidemiology Group, Department of Public Health Sciences, Karolinska \\ Institute, Norrbacka, SE-171 76 Stockholm, Sweden: ${ }^{2}$ Division of Epidemiology, Stockholm Centre of Public Health, \\ Norrbacka, SE-171 76 Stockholm, Sweden: ${ }^{3}$ Research Unit for Dietary Studies and Danish Epidemiology Science \\ Centre at the Institute of Preventive Medicine, Copenhagen University Hospital, Øster Søgade 18, 1399 \\ Copenhagen, Denmark
}

Submitted 11 July 2005: Accepted 14 December 2005

\begin{abstract}
Objective: The aim of the present study was to examine the relationships between genetic susceptibility to obesity, physical activity (PA), dietary fibre, sugar and fat intakes and 4-year changes in body mass index (BMI) and attained waist circumference (WC) in a cohort of 287 monozygotic and 189 dizygotic young adult male twin pairs. Increased knowledge about interactions between genes and environment may provide insight into why some individuals are more prone to obesity than others.

Design: Information about PA, BMI, dietary habits, WC and potential confounders was collected by questionnaire in 1998 and 2002. The cohort data were analysed by mixed linear models.

Results: Twins with low PA attained larger WC than twins with high PA (difference $2.5 \mathrm{~cm}$; 95\% confidence interval (CI) 1.3, 3.6). The twins with the lowest fibre intake were found to have attained the highest WC and to have increased most in BMI (difference between highest and lowest fibre intakes: $1.6 \mathrm{~cm}, 95 \%$ CI $0.4,2.9$ and $0.45 \mathrm{~kg} \mathrm{~m}^{-2}, 95 \%$ CI $0.15,0.76$, respectively). Furthermore, our results suggested the presence of interactions so that twins with genetic susceptibility to obesity were more prone to have larger WC if sedentary than twins without genetic susceptibility.

Conclusion: PA and a diet rich in fibre may be protective against weight gain among younger adult men. An interaction between PA, genes and attained WC is a novel finding which needs confirmation by other studies.
\end{abstract}

Keywords

Gene-environment interaction Physical activity

Diet

Waist circumference Body weight
A large number of studies have shown that genes as well as environmental factors are important in the aetiology of obesity. Heterogeneity of time trends has been reported in some studies and this may indicate variations in susceptibility to obesity among population subsets ${ }^{1}$.

No consistent evidence has been presented for an association between physical activity (PA) and subsequent weight change $\mathrm{e}^{2}$. Convincing evidence is also lacking for a role of intake of fibre, sugars and fat in weight change ${ }^{3}$. It is a limitation of most previous studies that genetic factors have not been taken into account. Knowledge about modifying effects of genes on relationships between PA, dietary intake and weight change may provide insight into why some people develop obesity while others do not.

Previous twin research indicates that genetic factors modify the effects of PA and diet on weight change. In genetically susceptible men, a sedentary lifestyle has been associated with larger weight increases than in men without such susceptibility ${ }^{4}$. A preference for fruits, vegetables and grain products, as well as intake of these food items, also seems to be partly genetically determined $^{5}$. With respect to weight change, a particular sensitivity to dietary fat has been reported among women with a familial predisposition to obesity ${ }^{6}$.

The aim of the present study was to examine the impact of PA, dietary fibre, sugar and fat intakes, genetic susceptibility and their interactions (gene-environment, $\mathrm{G} \times \mathrm{E}$ ) on change in body mass index (BMI) and attained waist circumference (WC), in a cohort of 287 monozygotic (MZ) and 189 dizygotic (DZ) young adult male twin pairs from Sweden.

\section{Materials and methods}

The Swedish Young Male Twins Register was created in 19987. All 3566 male twins born in Sweden from 1973 to 
1979, who were Swedish residents in 1997, were included. Of the 3566 twins, 2810 (79\%) answered a mailed questionnaire in 1998 focusing on PA, and 2169 (61\% of all) also responded to a second questionnaire in 2002 requesting information on $\mathrm{PA}$ and dietary habits. The Ethics Committee at the Karolinska Institute, Stockholm, Sweden, has approved this study.

In the present study we excluded 475 individuals who did not have complete data on the variables used, 13 individuals with severe diseases which could affect their level of PA (e.g. amputations and cerebral pares) or diet (e.g. Crohn's disease), 309 individuals of undetermined zygosity, and 420 individuals who belonged to incomplete pairs. After these exclusions, $287 \mathrm{MZ}$ and $189 \mathrm{DZ}$ twin pairs remained in the final study population.

The information about zygosity collected by the questionnaires in 1998 and 2002 was based on validated questions of similarity in childhood and difficulties teachers might have had in distinguishing between the twins $^{8,9}$. In 2003, twins belonging to complete pairs of undetermined zygosity were offered a DNA test. DNA was purified from buccal cells collected by mouth washing, and zygosity was determined by 16 highly polymorphic microsatellite markers as described elsewhere ${ }^{10,11}$. In this study population 65 additional pairs were classified as MZ or DZ by the DNA testing.

\section{Outcome variables}

The twins were asked to report their height and weight in 1998 and 2002. Based on self-reported data, BMI was calculated as weight divided by the square of height $\left(\mathrm{kg} \mathrm{m}^{-2}\right)$. In 2002 the twins were asked to measure their WC midway between the lower border of the chest and the anterior iliac crest with a tape measure that was mailed together with the questionnaire. An illustration helped them to perform this task properly. Outcome variables were the change in BMI between 1998 and 2002 and the WC measured in 2002.

\section{Explanatory variables}

The following questions were asked about PA in the 1998 and 2002 questionnaires: 'How would you describe your leisure-time PA during the last 12 months?' and 'How would you describe your occupational physical activity during the last 12 months?' Responses were 'sedentary/light exercise (not sweating)', 'medium exercise (sweating)' and 'hard exercise (sweating and out of breath)'. In the questionnaire 2002 the validated Baecke instrument was additionally included $^{12,13}$. Rank correlation coefficients were calculated between each of the two questions of leisure-time PA and occupational PA and the relevant dimensions of the Baecke instrument. The correlations were 0.83 (95\% confidence interval (CI) $0.81,0.84$ ) and 0.85 (95\% CI 0.83, 0.86), respectively. Consequently we decided to use the global questions of PA in both 1998 and 2002 as they seemed to give reasonable valid information.
PA was dichotomised into high (medium and hard) and low (sedentary and light); this was due to few observations in the longitudinal extreme categories. Leisure-time PA was stratified by engagement in body-building activities in some of the analyses described below. Body-building activities were defined as sport activities known to increase muscle mass extensively, e.g. weight lifting. In the $\mathrm{G} \times \mathrm{E}$ interaction analyses we were not able to use the longitudinal PA categorisation due to too few observations. In these analyses PA from either 1998 or 2002 was used. When WC in 2002 was the outcome variable, PA from the same time point was used. When, on the other hand, the BMI difference between 1998 and 2002 was the outcome variable, PA in 1998 was used as explanatory variable.

The following question was asked in 2002 about diet: 'How many days the last week have you been eating the following foods; fruit, vegetables, French fries, pizza, crisps, soda, chocolate/candy, coarse rye bread?' An additional question about shortening was included: 'How much shortening do you use on your bread?' This question was accompanied by an illustration showing four pieces of bread with increasing amount of shortening. Dietary indices were constructed by dividing the food items into the following three groups: rich in fibre (fruit, vegetables and coarse rye bread), rich in fat (French fries, pizza and crisps) and rich in sugar (soda and chocolate/candy). For each of these groups an index was constructed which contained the number of days per week the foods items had been consumed. Each of these indices was divided into three categories: high (upper 20\%), intermediate (intermediate 60\%) and low (lower 20\%). The question about shortening was dichotomised into thick layer and thin layer and was used independently as a separate indicator of fat intake.

\section{Potential confounding factors assessed in 2002}

Information about education level was dichotomised into high education (more than 12 years of education) and low education (12 years of education or less). Contact frequency among twin brothers was dichotomised into often contact (several times a week or more) and less often contact (once a week or less). Information on smoking was dichotomised into smokers (now and then/regularly) and non-smokers. The following questions were posed about alcohol consumption: 'How often do you drink alcohol?' and 'How much on each occasion?', and included beer, wine, liqueurs and spirits which allowed us to calculate alcohol consumption in grams per week ${ }^{14}$. Consumption was divided into four categories: very high $\left(>210\right.$ g week $\left.^{-1}\right)$, high $\left(60-210\right.$ g week $\left.^{-1}\right)$, intermediate $\left(10-59\right.$ g week $\left.^{-1}\right)$ and low $\left(<10\right.$ g week $\left.^{-1}\right)$.

\section{Statistical analysis}

The outcome variables were change in BMI between 1998 and 2002 and the attained WC. First, we calculated 
unadjusted means of the outcome variables. Second, we estimated mean differences for change in BMI and attained WC in 2002 between a reference category and each of the other categories of food intake and PA variables by mixed linear regression models ${ }^{15}$ using the MIXED PROCEDURE in SAS ${ }^{16}$. The full model included adjustments for physical activity in 1998 and 2002, intake of dietary fibre, age, baseline BMI, education, contact between the twin brothers, occupational physical activity, dietary fat, amount of shortening on bread, alcohol consumption and smoking.

The outcomes, change in BMI between 1998 and 2002 and the attained WC, are correlated within the twin pairs due to shared genes and environment and these withinpair correlations are stronger for MZ than for DZ twins. This violates the assumption of independent observations needed in standard regression models and can lead to biased estimates if not taken into account. When estimating the model parameters with the mixed model, this is accounted for by incorporating one random intercept for each twin pair and one random residual component for $\mathrm{MZ}$ twins and $\mathrm{DZ}$ twins, respectively ${ }^{15}$. Residual intraclass correlations can be derived from the estimated random components ${ }^{15,17}$ and a larger residual intraclass correlation for MZ than for DZ is evidence of genetic influence on the phenotype ${ }^{18}$. Confidence intervals for the fixed parameters were derived by Wald statistics. Approximate 95\% CI for the intraclass correlations and corresponding differences were calculated using Fisher's z-transformation.

Further analyses explored if associations between PA, food intake and attained WC or BMI change were modified by genetic susceptibility to increased WC/BMI change. We used the information obtained from the fitted mixed linear models to classify genetic susceptibility for obesity; a method slightly modified after Samaras et al. ${ }^{19}$. From the models each individual has a predicted attained WC and BMI change but will diverge more or less from the predicted values; the model residuals. These residuals can be said to represent the genetic factors and environmental factors not taken into account in the model. If both twins had residuals below the median they were classified as belonging to a low-risk group, if both belonged to the upper half they were classified to a high-risk group, and if twins were discordant they were excluded from the analysis. Discordant pairs were excluded to minimise misclassification bias. Had all MZ twins been included, some twin brothers, who share all their genes and therefore have identical genetic risk, could have been classified to different risk groups.

We analysed the associations between PA, fibre consumption and fat consumption and attained WC/BMI change by mixed linear models stratified by genetic susceptibility for obesity. Because the twins had been classified in high-risk and low-risk groups after adjustment for other measures of health behaviour in the fully adjusted mixed linear model mentioned above, no further adjustments were made in the $\mathrm{G} \times \mathrm{E}$ interaction analyses. Differences in strengths of associations between PA, fibre consumption and fat consumption and attained WC/BMI change over strata of genetic risk would indicate $\mathrm{G} \times \mathrm{E}$ interactions.

\section{Results}

A total of $287 \mathrm{MZ}$ and $189 \mathrm{DZ}$ young adult male twin pairs were included in the main analyses. The 952 subjects included in the analyses were compared with the 2526 subjects not included in the analysis, using birth date, BMI from the conscription examination at age 18 years and educational level from the 1998 survey. BMI was available for $94 \%$ and $89 \%$ of subjects included and not included, respectively, and education for $100 \%$ and 68\%, respectively. Twins included were on average born 10 days earlier than the twins not included $(P=0.72)$ and did not differ with respect to BMI (difference $0.05 \mathrm{~kg} \mathrm{~m}^{-2}, P=0.67$ ) at age 18 years. However, there was a larger proportion of highly educated subjects (12 years of education or more) among the twins included than among those not included, $43.1 \%$ and $34.6 \%$ respectively $(P=0.0003)$. The participating MZ and DZ twins did not differ statistically with regard to age, BMI or WC (Table 1 ).

In our univariate analyses, subjects with low levels of leisure-time PA in both 1998 and 2002 had somewhat larger WC and larger gain in BMI between 1998 and 2002 than those with high levels of leisure-time PA (Table 2). As described above, individuals with high leisure-time PA in 2002 were stratified by engagement in body-building

Table 1 Mean age and measures of body size among $574 \mathrm{MZ}$ and $378 \mathrm{DZ}$ twins

\begin{tabular}{|c|c|c|c|}
\hline Age and body measures in 1998 and 2002 & MZ twins & DZ twins & $\begin{array}{c}P \text {-value for } \\
\text { difference }(t \text {-test) }\end{array}$ \\
\hline Age in 2002 (years) & $25.6(0.11)$ & $25.8(0.14)$ & 0.26 \\
\hline $\mathrm{BMI}$ in $1998\left(\mathrm{~kg} \mathrm{~m}^{-2}\right)$ & $22.7(0.14)$ & $23.0(0.17)$ & 0.11 \\
\hline BMI in $2002\left(\mathrm{~kg} \mathrm{~m}^{-2}\right)$ & $23.5(0.15)$ & $24.0(0.19)$ & 0.07 \\
\hline WC in $2002(\mathrm{~cm})$ & $85.2(0.43)$ & $86.3(0.53)$ & 0.10 \\
\hline BMI change between 1998 and $2002\left(\mathrm{~kg} \mathrm{~m}^{-2}\right)$ & $0.8(0.07)$ & $0.9(0.09)$ & 0.51 \\
\hline
\end{tabular}

MZ - monozygotic; DZ - dizygotic; BMI - body mass index; WC - waist circumference.

Data are expressed as mean (standard error). 
Table 2 Effects of PA, eating habits, alcohol consumption and smoking on WC in 2002 and BMI change between 1998 and 2002 among 952 twins

\begin{tabular}{|c|c|c|c|}
\hline Independent variable & $n$ & WC in $2002(\mathrm{~cm})$ & $\begin{array}{l}\text { BMI change between } \\
1998 \text { and } 2002\left(\mathrm{~kg} \mathrm{~m}^{-2}\right)\end{array}$ \\
\hline \multicolumn{4}{|c|}{ Leisure-time PA in 1998 and $2002^{*}$} \\
\hline Low-Low & 185 & $87.9(10.3)$ & $1.00(1.66)$ \\
\hline High-Low & 66 & $86.3(9.1)$ & $0.90(1.53)$ \\
\hline Low-High: B & 56 & $85.9(9.4)$ & $1.07(1.74)$ \\
\hline Low-High: No B & 122 & $86.2(9.5)$ & $0.91(1.75)$ \\
\hline High-High: B & 265 & $84.4(6.6)$ & $0.89(1.45)$ \\
\hline High-High: No B & 258 & $84.7(7.2)$ & $0.67(1.24)$ \\
\hline \multicolumn{4}{|c|}{ Occupational PA in 1998 and 2002} \\
\hline Low-Low & 483 & $84.9(8.3)$ & $0.74(1.42)$ \\
\hline Low-High & 76 & $85.9(8.9)$ & $1.18(1.66)$ \\
\hline High-Low & 171 & $85.3(7.6)$ & $0.90(1.42)$ \\
\hline High-High & 222 & $87.3(8.7)$ & $1.00(1.62)$ \\
\hline \multicolumn{4}{|l|}{ Dietary fibre } \\
\hline Low & 173 & $86.7(10.3)$ & $1.18(1.73)$ \\
\hline Intermediate & 568 & $85.7(8.1)$ & $0.83(1.45)$ \\
\hline High & 211 & $84.4(7.1)$ & $0.71(1.37)$ \\
\hline \multicolumn{4}{|l|}{ Dietary fat } \\
\hline Low & 120 & $85.9(7.6)$ & $0.80(1.78)$ \\
\hline Intermediate & 452 & $86.2(8.9)$ & $0.92(1.49)$ \\
\hline High & 380 & $84.8(7.8)$ & $0.83(1.40)$ \\
\hline \multicolumn{4}{|l|}{ Dietary sugar } \\
\hline Low & 134 & $86.1(7.8)$ & $0.66(1.54)$ \\
\hline Intermediate & 584 & $85.6(8.3)$ & $0.88(1.47)$ \\
\hline High & 234 & $85.3(8.8)$ & $0.95(1.52)$ \\
\hline \multicolumn{4}{|l|}{ Shortening on bread } \\
\hline Thick layer & 237 & $85.5(9.4)$ & $0.83(1.47)$ \\
\hline Thin layer & 715 & $85.7(8.0)$ & $0.88(1.50)$ \\
\hline \multicolumn{4}{|l|}{ Alcohol consumption } \\
\hline Low & 86 & $85.0(10.1)$ & $0.68(1.67)$ \\
\hline Intermediate & 391 & $85.5(8.4)$ & $0.93(1.50)$ \\
\hline High & 434 & $85.9(8.0)$ & $0.87(1.46)$ \\
\hline Very high & 41 & $84.7(7.0)$ & $0.62(1.40)$ \\
\hline \multicolumn{4}{|l|}{ Smoking } \\
\hline Yes, regularly & 55 & $87.4(10.0)$ & $0.94(2.01)$ \\
\hline Now and then & 108 & $86.1(8.2)$ & $0.85(1.42)$ \\
\hline No & 789 & $85.4(8.3)$ & $0.87(1.46)$ \\
\hline
\end{tabular}

PA - physical activity; WC - waist circumference; BMI -body mass index.

Data are expressed as unadjusted mean (standard deviation).

* Leisure-time PA stratified by body-building (B) for those with high PA level in 2002.

activities because these activities increase muscle mass. Individuals engaged in body-building increased slightly more in BMI than those not engaged in such activities. Of the young men with high levels of leisure-time PA in 2002, $36 \%$ were involved in body-building activities. Subjects with low intake of fibre in 2002 had a tendency towards higher attained WC and increased BMI change than those with high intake of fibre (Table 2).

The multivariate model included baseline BMI, leisuretime PA, occupational PA, dietary fibre, dietary fat, dietary sugar, amount of shortening, alcohol consumption, smoking, education and contact between twin brothers. In the fully adjusted models, a significant difference in attained WC between those who were sedentary in 1998 and 2002 and those active both years and not engaged in body-building activities $(2.5 \mathrm{~cm}, 95 \%$ CI 1.2, 3.6; $P<0.001$ ) became evident (Table 3 ). This difference was not evident without stratification by body-building activities. No consistent association between PA and change in BMI was seen (Table 3). Twins who consumed a diet low in fibre had a history of greater BMI increase than those who consumed a diet rich in fibre (difference $0.45 \mathrm{~kg} \mathrm{~m}^{-2}, 95 \%$ CI $0.15,0.76 ; P=0.004$ ). Accordingly, those who consumed a diet low in fibre also had larger WC than those who consumed a diet rich in fibre (difference $1.6 \mathrm{~cm}, 95 \%$ CI $0.4,2.8 ; P=0.010$ ). We found no effects of smoking, alcohol consumption, dietary fat, dietary sugar or occupational PA on change of BMI or attained WC (data not shown).

When all independent variables in the fully adjusted models had been taken into account, substantial genetic effects on BMI change and attained WC were evident. For attained WC the residual intraclass correlations were 0.28 among DZ twins and 0.46 among MZ twins, and the difference was 0.18 (95\% CI 0.00, 0.37). For BMI change the residual intraclass correlations were 0.12 for $\mathrm{DZ}$ and 0.44 for MZ and the difference was 0.32 (95\% CI $0.13,0.50)$.

We performed six separate analyses for $G \times E$ interaction: PA, dietary fibre and dietary fat were 
Table 3 Adjusted effects of PA and dietary fibre on WC in 2002 and BMI change between 1998 and 2002 among 952 twins*

\begin{tabular}{lccc}
\hline Independent variable & $n$ & WC in 2002 $(\mathrm{cm})$ & $\begin{array}{c}\text { BMI change between } \\
1998 \text { and } 2002\left(\mathrm{~kg} \mathrm{~m}^{-2}\right)\end{array}$ \\
\hline $\begin{array}{l}\text { Leisure-time PA in 1998 and 2002† } \\
\text { Low-Low }\end{array}$ & 185 & $2.5(1.3,3.6)$ & \\
High-Low & 66 & $1.2(-0.1,2.5)$ & $0.21(-0.08,0.50)$ \\
Low-High: B & 56 & $0.8(-0.8,2.4)$ & $0.18(-0.14,0.50)$ \\
Low-High: No B & 122 & $1.3(-0.4,3.0)$ & $0.22(-0.18,0.62)$ \\
High-High: B & 265 & $-0.8(-1.8,0.2)$ & $0.15(-0.27,0.58)$ \\
High-High: No B & 258 & 0 & $0.19(-0.06,0.45)$ \\
Dietary fibre & 173 & $1.6(0.4,2.8)$ & $0.45(0.15,0.76)$ \\
Low & 568 & $1.2(0.3,2.2)$ & $0.17(-0.06,0.40)$ \\
Intermediate & 211 & 0 & 0 \\
High & & & \\
\hline
\end{tabular}

PA - physical activity; WC - waist circumference; BMI -body mass index.

Data are expressed as mean difference ( $95 \%$ confidence interval).

* Mean differences from reference levels estimated by mixed linear models and adjusted for all independent variables in the table and additionally adjusted for age, baseline BMI, education, contact between the twin brothers, occupational PA, dietary fat, amount of shortening on bread, alcohol consumption and smoking.

† Leisure-time PA stratified by body-building (B) for those with high PA level in 2002.

analysed in relation to WC (analyses including $207 \mathrm{MZ}$ pairs) and BMI change between 1998 and 2002 (analyses including $180 \mathrm{MZ}$ pairs). PA and fibre intake were considered because significant associations were found in the fully adjusted mixed linear models (Table 3 ) and fat intake because $\mathrm{G} \times \mathrm{E}$ interactions have been previously reported ${ }^{6}$. Figure 1 shows the results from the $\mathrm{G} \times \mathrm{E}$ analyses. If the difference in $\mathrm{WC} / \mathrm{BMI}$ change is different among those with genetic susceptibility (high-risk group) for obesity compared with those without such susceptibility (low-risk group), this would imply that genetics is interacting with the environmental factors. The association between physical activity and WC/BMI change was different for those genetically predisposed to obesity compared with those in the lowrisk group. The difference between sedentary and active MZ twins, with respect to WC measured in 2002, was $2.5 \mathrm{~cm}(95 \%$ CI $0.3,4.8 ; P=0.028)$ larger among those
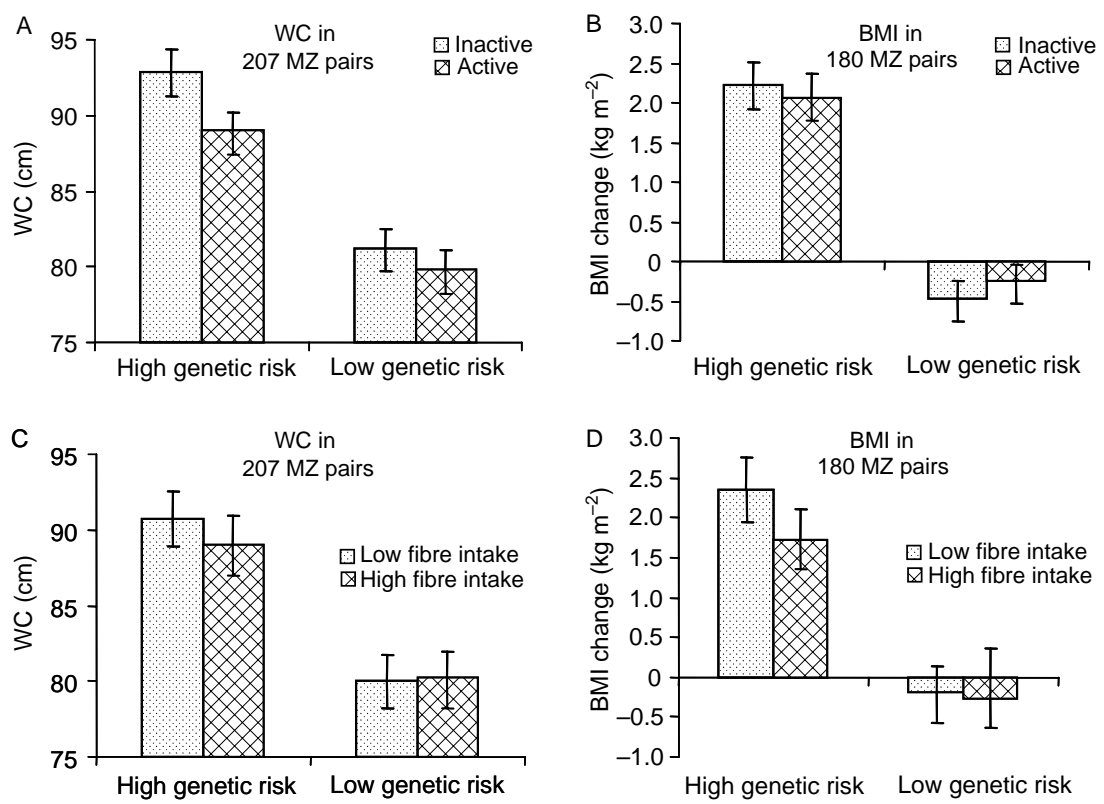

Fig. 1 Gene-environment $(G \times E)$ interactions. Means and 95\% confidence intervals (shown by vertical bars) were estimated by mixed linear models. Analyses among monozygotic (MZ) twins were stratified by genetic susceptibility for obesity. If physical activity and/or a diet rich in fibre affects those with genetic susceptibility for obesity differently than those without susceptibility, this would imply $\mathrm{G} \times \mathrm{E}$ interactions. (A) shows that attained waist circumference (WC) is significantly more affected by physical activity for individuals with high susceptibility for obesity than for those with low genetic susceptibility for obesity. (B) shows that change in body mass index (BMI) is nonsignificantly more affected by physical activity for individuals with high susceptibility for obesity than for those with low genetic susceptibility for obesity. (C) shows a non-significant larger difference in WC among those with high genetic susceptibility for obesity than among those with low genetic susceptibility for obesity. (D) shows a non-significant larger difference in BMI change among those with high genetic susceptibility for obesity than among those with low genetic susceptibility for obesity 
with high genetic risk for obesity than among those with low genetic risk. Similar results were observed for BMI change but the difference between sedentary and active MZ twins was not significantly larger among those with high genetic risk for obesity than among the low risk individuals, $0.38 \mathrm{~kg} \mathrm{~m}^{-2}$ (95\% CI $\left.-0.11,0.87 ; P=0.13\right)$. The association between dietary fibre and excess weight was not significantly different for those genetically predisposed to obesity compared with the low-risk group. There was a non-significant larger difference in BMI change and in WC among high-risk than among low-risk twins with low and high dietary fibre intake. Differences between the associations were estimated to be $0.26 \mathrm{~kg} \mathrm{~m}^{-2}(95 \% \mathrm{CI}-0.44,0.96 ; P=0.47)$ for $\mathrm{BMI}$ change and $2.0 \mathrm{~cm}(95 \% \mathrm{CI}-1.3,5.2 ; P=0.24)$ for attained WC. Differences between the associations between fat intake and WC and BMI change for high and low genetic risk for obesity were estimated to $0.3 \mathrm{~cm}$ (95\% CI $-0.5,1.0 ; P=0.46)$ and $0.77 \mathrm{~kg} \mathrm{~m}^{-2}(95 \% \mathrm{CI}$ $-0.17,1.72 ; P=0.11$ ), respectively. $\mathrm{G} \times \mathrm{E}$ analyses of the DZ twins supported the MZ twins' results but confidence intervals were wider (data not shown).

\section{Discussion}

In this study we have shown the importance of leisuretime PA and a diet rich in fibre for attained WC in particular, and to some extent for BMI change. Twins with a diet rich in fibre in 2002 increased less in BMI and attained lower WC than those with a diet poor in fibre in 2002. However, contrary to other investigators, we found no association between high intake of fat or sugar and subsequent BMI change or attained $\mathrm{WC}^{6}$. In accordance with another study ${ }^{4}$, we found higher residual intraclass correlations for change in BMI and attained WC among MZ than DZ twins, recognised to be due to genetic factors ${ }^{18}$.

Our results showed the presence of interactions between genetic susceptibility to obesity and PA. Twins with genetic susceptibility to obesity were more prone to larger WC if sedentary than twins without such susceptibility. An interaction between PA, genes and attained WC is a novel finding, not previously reported in the literature. Although not statistically significant, our results also showed a tendency towards an interaction between dietary intake of fibre and genes with regard to both BMI change and attained WC. However, unlike a previous Swedish family study which showed an increased genetic susceptibility to weight gain from a high fat intake among a subset of females ${ }^{6}$, but in accordance with others ${ }^{19}$, our results did not indicate $\mathrm{G} \times \mathrm{E}$ interactions between fat intake and BMI change or attained WC. A potential explanation for these inconsistencies might be sex differences or measurement errors $^{20,21}$

In this cohort of young men, occupational PA was not significantly associated with BMI change or attained WC.
We believe that the occupational dimension of PA may be more informative in an older cohort with more clearly established and dispersed roles in working life. There might also be different mechanisms driving the motivation for physical activity in leisure time and occupational physical activity, which might be an explanation for why the results differ.

This study has several strengths. First, the longitudinal twin design created possibilities for assessment of $\mathrm{G} \times \mathrm{E}$ interactions in relation to BMI change that would not have been conceivable in cohort studies on singletons without extensive family information. Hence, the matched nature of $M Z$ twin pairs made it possible for us to explore relationships between PA and BMI change/attained WC with control for genetic factors. Second, our study measured PA and dietary habits concurrently. A third strength was the ability to adjust for potential confounding factors, e.g. educational level, smoking habits and alcohol consumption. A fourth strength is related to the methods used for measurement of PA. In our 2002 questionnaire, Baecke's method for assessment of PA was applied ${ }^{12}$. This method has been shown to have high validity compared with the gold standard method of doubly labelled water ${ }^{13}$. In the present study the relevant dimensions of the Baecke questionnaire correlated strongly with our global questions of leisure-time $\mathrm{PA}$ and occupational PA, indicating that these questions provided us with good measures of the PA in this cohort of twins.

The study also has limitations. A longer follow-up period would have created more variability in BMI changes, and thus potentially a higher probability of detecting effects. The need for more statistical power was particularly evident in the $\mathrm{G} \times \mathrm{E}$ interaction analyses. Our frequency questions on dietary intake did not allow us to assess total daily energy intake, and it is thus a limitation that our indices of intake of fibre, fat and simple carbohydrates could not be adjusted for total energy. Owing to the questionnaire design of this study, data on body size, PA and dietary intakes are selfreported. While non-differential misclassification of exposure and outcome variables is likely to attenuate measures of association, the consequences of differential misclassification are more difficult to assess. It is, however, possible that our study subjects overreported or underreported to the same extent in 1998 and 2002. If this is true, it is less likely that differentially biased estimates of changes in BMI would have been obtained. However, non-differential effects would of course persist. As described above, the fitted models were adjusted for a range of behavioural and social factors with the goal to achieve unbiased estimates of associations between PA and dietary factors and attained WC/BMI change. Residual confounding cannot, however, be entirely ruled out. We chose an approach that we have modified slightly after Samaras et al. for 
estimating $\mathrm{G} \times \mathrm{E}$ interactions ${ }^{19}$. Twins were classified to high or low genetic susceptibility using the residuals from the fully adjusted mixed linear models. We excluded MZ twins in pairs who differed in classification from the $\mathrm{G} \times \mathrm{E}$ analyses since they, by definition, have the same genetic risk. Last, our study has some inevitable limitations with respect to generalisability since it included young adult males only. However, the present study results agree with previous results on Finnish twins, which showed an interaction between genes and PA in relation to weight change among males, aged 18-40 years, but not among females ${ }^{4}$.

As reported in our analyses of non-participation, study subjects were similar to non-participants with the exception of education level, which was slightly higher among participants. Owing to the population-based and nationwide nature of our study, and the fact that twins have repeatedly been shown not to differ from singletons with respect to health or health-related traits ${ }^{22}$, we believe that our results are representative for young men in Sweden born in the 1970s.

\section{Public bealth implications}

Although the associations between PA and the change in BMI or attained WC are modest they are none the less important. Obesity is a chronic disease that develops over a long period of time and small changes in BMI or WC in young adulthood may have serious health consequences in later life. Based on the present findings, and in accordance with a recent publication of the World Health Organization, it is suggested that future nutritional recommendations focus more on promoting a higher intake of dietary fibre and less on lowering dietary fat ${ }^{23}$.

A recent consensus statement by the International Society for the Study of Obesity recommends at least 45-60 minutes of moderate PA activity per day to prevent weight gain among adults ${ }^{24}$. Due to heterogeneity in susceptibility to weight gain ${ }^{1,25}$ and putative modifying effects of genes on the relationship between PA and weight change, 45 minutes of moderate PA may be enough to prevent weight gain in some, whereas 60 minutes might be too little in others.

\section{Conclusions}

PA and a diet rich in fibre may be protective against weight gain. The interaction between PA, genetic susceptibility and WC is a novel finding, not previously reported in the literature. However, further follow-up of the present Swedish twin study and results from other populationbased family and twin studies are needed before more specific recommendations adapted to genetic risks can be developed and proposed.

\section{Acknowledgements}

The authors would like to thank the Swedish Council for Working Life and Social Research (contract number 20020623) for their financial support of this study.

\section{References}

1 Heitmann BL, Stroger U, Mikkelsen KL, Holst C, Sørensen TIA. Large heterogeneity of the obesity epidemic in Danish adults. Public Health Nutrition 2004; 7: 453-60.

2 Fogelholm M, Kukkonen-Harjula K. Does physical activity prevent weight gain - a systematic review. Obesity Reviews 2000; 1: 95-111.

3 Togo P, Osler M, Sørensen TIA, Heitmann BL. A longitudinal study of food intake patterns and obesity in adult Danish men and women. International Journal of Obesity and Related Metabolic Disorders 2004; 28: 583-93.

4 Heitmann BL, Kaprio J, Harris JR, Rissanen A, Korkeila M, Koskenvuo M. Are genetic determinants of weight gain modified by leisure-time physical activity? A prospective study of Finnish twins. American Journal of Clinical Nutrition 1997; 66: 672-8.

5 Heitmann BL, Harris JR, Lissner L, Pedersen NL. Genetic effects on weight change and food intake in Swedish adult twins. American Journal of Clinical Nutrition 1999; 69: 597-602.

6 Heitmann BL, Lissner L, Sørensen TIA, Bengtsson C. Dietary fat intake and weight gain in women genetically predisposed for obesity. American Journal of Clinical Nutrition 1995; 61 : 1213-7.

7 Rasmussen F, Johansson-Kark M. The Swedish Young Male Twins Register: a resource for studying risk factors for cardiovascular disease and insulin resistance. Twin Research 2002; 5: 433-5.

8 Cederlöf R, Friberg L, Jonsson EKL. Studies on similarity diagnosis on twins with the aid of mailed questionnaires. Acta Geneticae Medicae et Germellologiae 1961; 11: 338-62.

9 Pedersen N, Lichtenstein P. The Swedish Twin Registry - a presentation. In: Smedby B, Lundberg I, Sørensen TIA, eds. Scientific Evaluation of the Swedish Twin Registry. Stockholm: Swedish Council for Planning and Coordination of Research, 2000; 15-44.

10 Heath EM, Morken NW, Campbell KA, Tkach D, Boyd EA, Strom DA. Use of buccal cells collected in mouthwash as a source of DNA for clinical testing. Archives of Pathology and Laboratory Medicine 2001; 125: 127-33.

11 Tholin S, Rasmussen F, Tynelius P, Karlsson J. Genetic and environmental influences on eating behavior: the Swedish Young Male Twins Study. American Journal of Clinical Nutrition 2005; 81: 564-9.

12 Baecke JA, Burema J, Frijters JE. A short questionnaire for the measurement of habitual physical activity in epidemiological studies. American Journal of Clinical Nutrition 1982; 36: 936-42.

13 Philippaerts RM, Lefevre J. Reliability and validity of three physical activity questionnaires in Flemish males. American Journal of Epidemiology 1998; 147: 982-90.

14 Wing JK, Babor T, Brugha TE, Burke J, Cooper JE, Giel R, et al. SCAN. Schedules for clinical assessment in neuropsychiatry. Archives of General Psychiatry 1990; 47: 589-93.

15 Guo G, Wang J. The mixed or multilevel model for behavior genetic analysis. Behavior Genetics 2002; 32: 37-49.

16 SAS Institute, Inc.. SAS/STAT User's Guide, Version 8. Cary, NC: SAS Institute, Inc., 1999.

17 Neale MC, Maes HH. Methodology for Genetic Studies of Twins and Families. Dordrecht: Kluwer Academic, 2004. 
18 Iachina M, Jorgensen B, Christensen K, Iachine I. Analysis of functional abilities for elderly Danish twins using GEE models. Twin Research 2002; 5: 289-93.

19 Samaras K, Kelly PJ, Chiano MN, Arden N, Spector TD, Campbell LV. Genes versus environment. The relationship between dietary fat and total and central abdominal fat Diabetes Care 1998; 21: 2069-76.

20 Korkeila M, Kaprio J, Rissanen A, Koskenvuo M. Consistency and change of body mass index and weight. A study on 5967 adult Finnish twin pairs. International Journal of Obesity and Related Metabolic Disorders 1995; 19: 310-7.

21 Harris JR, Tambs K, Magnus P. Sex-specific effects for body mass index in the new Norwegian twin panel. Genetic Epidemiology 1995; 12: 251-65.
22 Evans DM, Martin NG. The validity of twin studies. GeneScreen 2000; 1: 77-9.

23 World Health Organization (WHO). Diet, Nutrition and the Prevention of Chronic Diseases. Report of the Joint WHO/Food and Agriculture Organization Expert Consultation. Geneva: WHO, 2003.

24 Saris WH, Blair SN, Van Baak MA, Eaton SB, Davies PS, Di Pietro L, et al. How much physical activity is enough to prevent unhealthy weight gain? Outcome of the IASO 1st Stock Conference and consensus statement. Obesity Reviews 2003; 4: 101-14.

25 Pietinen P, Vartiainen E, Mannisto S. Trends in body mass index and obesity among adults in Finland from 1972 to 1992. International Journal of Obesity and Related Metabolic Disorders 1996; 20: 114-20. 